\title{
Urine microRNAs as biomarkers for bladder cancer: a diagnostic meta-analysis
}

This article was published in the following Dove Press journal:

OncoTargets and Therapy

II August 2015

Number of times this article has been viewed

\section{Yidong Cheng* \\ Xiaheng Deng* \\ Xiao Yang* \\ Pengchao Li \\ Xiaolei Zhang \\ Peng Li \\ Jun Tao \\ Qiang Lu \\ Zengjun Wang}

Department of Urology, The First Affiliated Hospital of Nanjing Medical University, Nanjing, People's Republic of China

*These authors contributed equally to this work
Correspondence: Qiang Lu; Jun Tao Department of Urology, The First Affiliated Hospital of Nanjing Medical University, 300, Guangzhou Road, Nanjing 210029, Jiangsu Province, People's Republic of China Tel +86 25 837| 0079

Email doctorlvqiang@sina.com; taojun.njmu@।39.com
Background: The diagnostic value of microRNA (miRNA) detection in patients with bladder cancer $(\mathrm{BCa})$ is controversial. We performed a diagnostic meta-analysis to evaluate current evidence on the use of miRNA assays to diagnose BCa.

Methods: We systematically searched PubMed, Embase, and Web of Science for studies published before March 31, 2015. The pooled sensitivity, specificity, positive and negative likelihood ratios, diagnostic odds ratio, and area under the curve (AUC) were calculated to evaluate the overall test performance. Subgroup analyses were used to explore the betweenstudy heterogeneity. Deeks' funnel plot asymmetry test was used to test publication bias. We applied the software of RevMan 5.2 and Stata 11.0 to the meta-analysis.

Results: A total of 23 studies from nine articles were included in the meta-analysis, with a total of 719 patients and 494 controls. The pooled sensitivity and specificity were 0.75 (95\% confidence interval [CI], 0.68-0.80) and 0.75 (95\% CI, 0.70-0.80), respectively. The pooled positive likelihood ratio was 3.03 (95\% CI, 2.50-3.67); negative likelihood ratio was 0.33 (95\% CI, 0.27-0.42); and diagnostic odds ratio was 9.07 (95\% CI, 6.35-12.95). The pooled AUC was 0.81 (95\% CI, 0.78-0.85). Subgroup analyses indicated that the multiple miRNAs assays and urine supernatant assays showed high accuracies in diagnosing $\mathrm{BCa}$.

Conclusion: The miRNA assays may serve as potential noninvasive diagnostic tool for the detection of $\mathrm{BCa}$. However, the clinical application of miRNA assays for $\mathrm{BCa}$ diagnosis still needs further validation by large prospective studies.

Keywords: microRNAs, bladder cancer, diagnostic accuracy, meta-analysis

\section{Introduction}

Bladder cancer $(\mathrm{BCa})$ is among the most common urological cancers, with more than 330,000 new cases each year and resulting in more than 130,000 deaths per year. ${ }^{1}$ The gold standards for the initial diagnosis of $\mathrm{BCa}$ are cystoscopy and biopsy. However, these methods are invasive, uncomfortable, and costly. ${ }^{2,3}$ Currently, cytology is the most widely used noninvasive diagnostic tool with median sensitivity (SEN) and specificity (SPE) values of 35\% and 94\%, respectively. ${ }^{4}$ Furthermore, bladder tumor antigen stat with a SEN of $57 \%-79 \%$ and SPE of $48 \%-95 \%$, NMP22 (nuclear matrix protein 22) with a SEN of $49.5 \%-65 \%$ and SPE of $40 \%-87.3 \%$, and telomerase with a SEN of $70 \%-100 \%$ and SPE of $60 \%-70 \%$ are the most common biomarkers for BCa diagnosis. ${ }^{5}$ These urinary markers have poor SPE and SEN for BCa diagnosis and cannot match the cytology tool. Therefore, the development of highly accurate biomarkers for the detection of $\mathrm{BCa}$ would benefit patients.

microRNAs (miRNAs) are nonprotein-coding RNAs that posttranscriptionally regulate gene expression. Altered expression of miRNAs plays an important role in the initiation and progression of various diseases, including cancer. Aberrant expression of miRNAs in $\mathrm{BCa}$ has been identified in an increasing number of studies. In addition, studies on the 
use of urine and blood-based miRNAs for the diagnosis of $\mathrm{BCa}$ have been published. ${ }^{6-8}$ Hence, the current miRNA profiling has presented potential biomarkers for $\mathrm{BCa}$ diagnosis.

The diagnostic accuracy of miRNA has been widely evaluated, and emerging evidence has presented promising results, but the possible application and validity of miRNAs in diagnosing $\mathrm{BCa}$ remains controversial. Studies on the use of miRNA as a BCa diagnostic tool indicated a wide range of values for SEN and SPE. Such variation may be the result of differences in the types of miRNA profiled and in the specimens tested. For example, Wang et $\mathrm{al}^{9}$ reported the diagnostic accuracy of miR-200a for BCa and obtained a high SEN of $100 \%$ and a low SPE of 52.6\% . However, Snowdon et al ${ }^{10}$ revealed a moderate SEN of $80 \%$ and a high SPE of $100 \%$ for the diagnostic accuracy of miR-125b and miR-126. In addition, the diagnostic accuracies of miRNA profiling between single and multiple miRNAs showed significant differences. Currently, no meta-analysis has been published on the diagnostic performance of miRNA assays for BCa. Therefore, we performed a meta-analysis to review and assess the overall diagnostic accuracy of miRNA assays for BCa diagnosis.

\section{Methods}

\section{Literature search}

An initial search was performed in PubMed, Medline, Embase, and Web of Science. The last search was updated on March 31, 2015. Search strings were performed for microRNA or miRNA, diagnosis or sensitivity or specificity, and bladder cancer. The search was limited to studies published in the English language. The reviewer also handsearched the references of all included studies to ensure that a comprehensive search strategy was used.

\section{Study selection}

Two reviewers independently assessed eligible publications. Any disagreement between the two reviewers was resolved through a discussion with a third reviewer. The inclusion criteria were as follows: 1) the patients with $\mathrm{BCa}$ were confirmed by histopathology, 2) the diagnosis of $\mathrm{BCa}$ involved the use of urine miRNA, and 3) both the SEN and SPE data were provided. The exclusion criteria were as follows: 1) review articles, seminar articles, and case reports; 2) limited data (number of cases and/or controls was not provided); and 3) BCa diagnosis involved the use of blood and tissue miRNA.

\section{Data extraction}

The following data were extracted by two reviewers independently from each study: name of the first author, publishing time, country, specimen, test method, internal control, cancer type, number of $\mathrm{BCa}$ patients and healthy controls, miRNA profiling, and SEN and SPE data.

\section{Quality assessment}

Quality assessment was performed for each included study by independent reviewers using the Quality Assessment of Diagnostic Accuracy Studies (QUADAS-2) tool. ${ }^{11}$ The assessment consisted of four key domains, including patient selection, index test, reference standard, and flow and timing. Each domain was assessed in terms of the risk of bias, and the first three domains were assessed in terms of applicability. Each domain was marked as "high", "low", or "unclear", which corresponded to high risk, low risk, and unclear, respectively.

\section{Data synthesis and statistical analysis}

The data of diagnostic accuracy were calculated for each study, as follows: SEN, SPE, positive likelihood ratio (PLR), negative likelihood ratio (NLR), diagnostic odds ratio (DOR), and their 95\% confidence intervals (CIs). The analysis of diagnostic accuracy was based on a summary receiver operating characteristic (SROC) curve and the area under curve (AUC) of the SROC. ${ }^{12}$ The $Q$ test and $I^{2}$ statistic were used to assess heterogeneity in SEN and SPE among the studies. A $P$-value of less than 0.1 for $Q$ test and a value larger than $50 \%$ for $I^{2}$ indicated substantial heterogeneity, and subsequently, the random-effect model was applied. ${ }^{13,14}$

In addition, we also investigated subgroup in our metaanalysis to evaluate potential sources of between-study heterogeneity. The publication bias was tested using Deeks' funnel plot asymmetry test. ${ }^{15}$ Quality assessment was performed using RevMan 5.2, and statistical calculations were performed using STATA 11.0.

\section{Results Study selection and description}

The flow graph of study selections is presented in Figure 1. A total of 215 potentially relevant articles were selected with an established search strategy. After a detailed evaluation, nine $\operatorname{articles}^{8-10,16-21}$ involving 23 studies were used for this metaanalysis (Table 1). The total number of patients and controls were 719 and 494, respectively. All studies used reverse transcription polymerase chain reaction (RT-PCR) or quantitative RT-PCR method to measure the expression of miRNAs in urine. RNU48, RNU44, and RNAU6 were used as internal controls. Of the nine included studies, four studies used urine sediment as specimens, two studies used urine supernatant, and the three other studies were based on voided urine. 


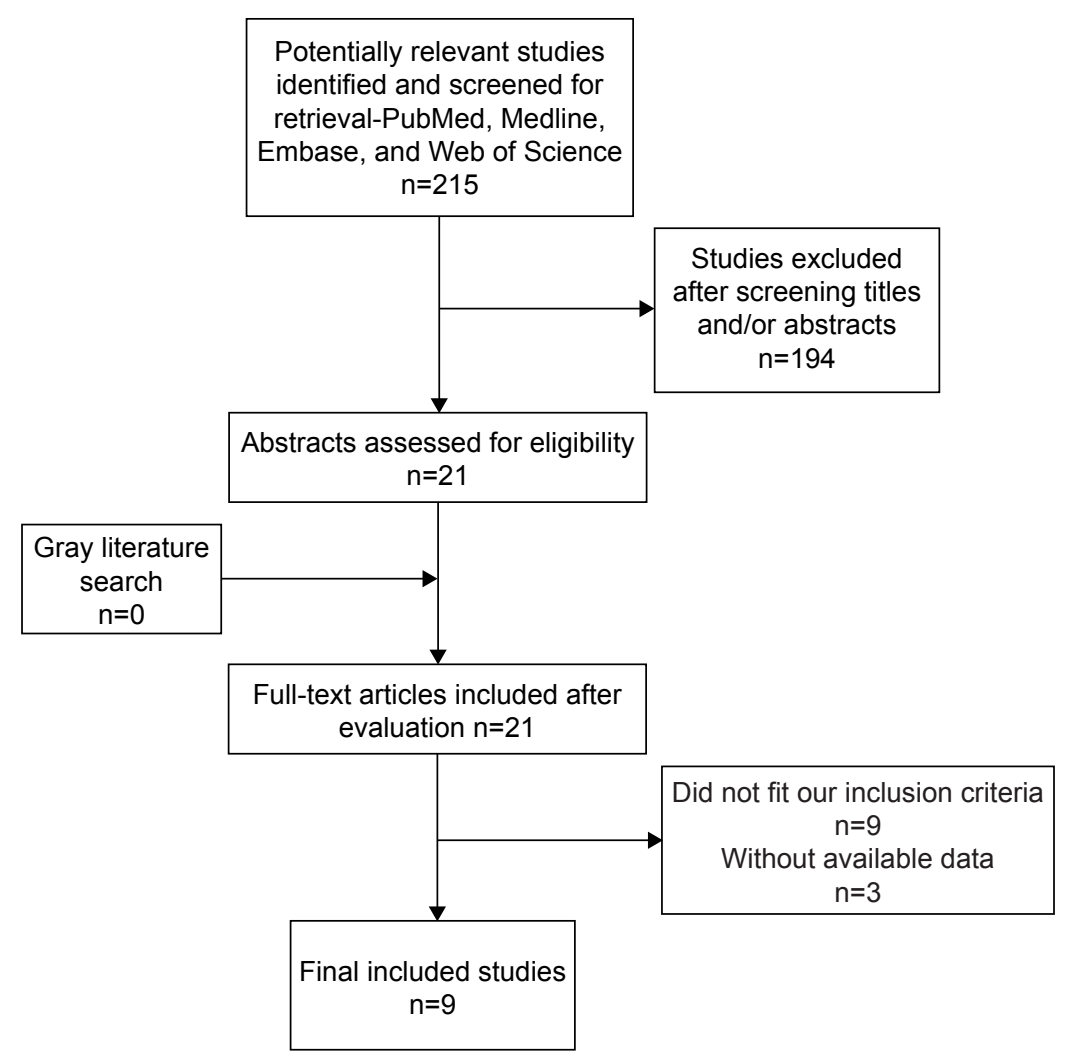

Figure I Flowchart of literature search and selection.

\section{Quality assessment of studies}

The outcomes of the QUADAS-2 study quality assessment are shown in a bar graph in Figure 2. The majority of all included studies in this review fulfilled most items in QUADAS-2, indicating that the overall quality of the included studies is generally good.

\section{Diagnostic accuracy}

The forest plot of SEN and SPE for miRNA assays is shown in Figure 3. Significant heterogeneity was found among the SEN and SPE results of the 23 studies because the $I^{2}$ for SEN is $80.24 \%$ (95\% CI, $72.74 \%-87.75 \%$ ), and the $I^{2}$ for SPE is $71.61 \%$ (95\% CI, $59.71 \%-83.59 \%)$. Therefore, this meta-analysis will apply a random-effect model to evaluate all parameters. The overall results of the miRNAs diagnostic accuracy are listed in Table 2. The SEN was 0.75 (95\% CI, $0.68-0.80)$, the SPE was 0.75 (95\% CI, 0.70-0.80), the PLR was 3.03 (95\% CI, 2.50-3.67), the NLR was 0.33 (95\% CI, 0.27-0.42), and the DOR was 9.07 (95\% CI, 6.35-12.95). The SROC curve for the 23 included studies is shown in Figure 4. The AUC of urine miRNA tests was 0.81 (95\% $\mathrm{CI}, 0.78-0.85)$, thereby implying a relatively high diagnostic accuracy.

\section{Subgroup analyses}

In this meta-analysis, we also performed subgroup analyses to identify potential sources of heterogeneity. Results of the diagnostic accuracy of subgroup analyses are listed in Table 2. For single miRNA profiling assays, SEN, SPE, and AUC values were 0.72 (95\% CI, 0.65-0.78), 0.74 (95\% CI, 0.69-0.79), and 0.79 (95\% CI, 0.76-0.83), respectively. For multiple miRNA profiling assays, the SEN, SPE, and AUC values were 0.86 (95\% CI, 0.78-0.91), 0.82 (95\% CI, 0.65-0.91), and 0.91 (95\% CI, 0.88-0.93), respectively. Multiple miRNA profiling was more accurate than single miRNA profiling.

Our meta-analysis assessed the diagnostic accuracy of miRNAs in different urine specimens. The pooled SEN, SPE, PLR, NLR, DOR, and AUC values of urine supernatant-based assay were $0.81,0.74,3.13,0.26,12.20$, and 0.84 , respectively. Meanwhile, the pooled SEN, SPE, PLR, NLR, DOR, and AUC values of voided urine-based assay were $0.77,0.76,3.12,0.31,9.99$, and 0.81 , respectively. Urine sediment-based assay presented a modest performance in $\mathrm{BCa}$ diagnosis (Table 2). Urine supernatant-based assay showed higher SEN than urine sediment-based assay. Thus, urine supernatant-based assay could be more reliable for clinical application than urine sediment-based assay. 


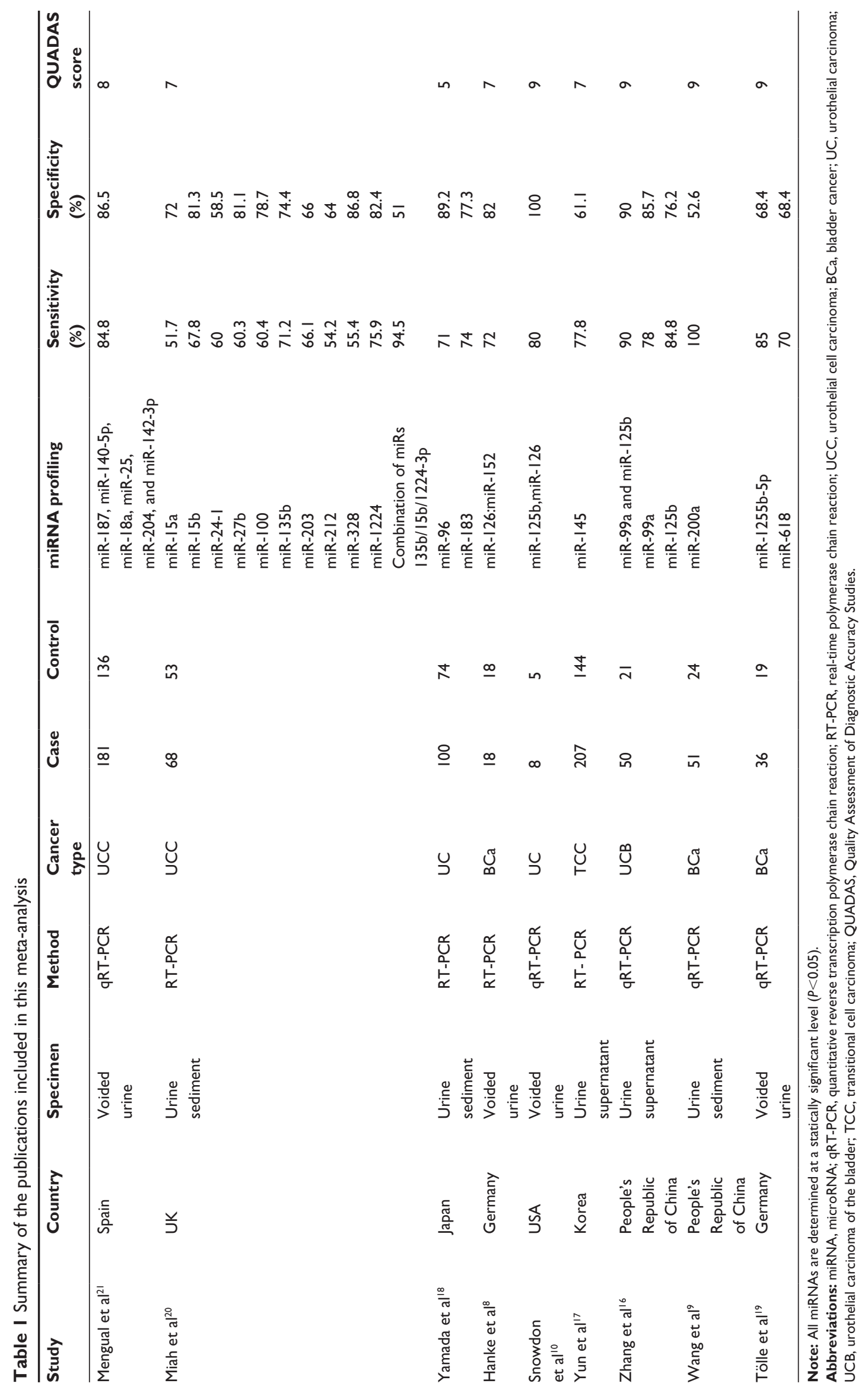




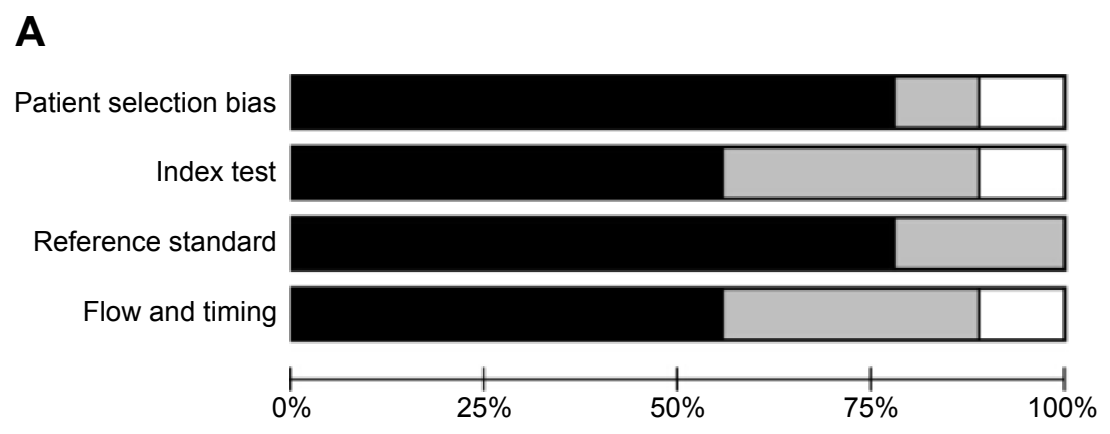

B

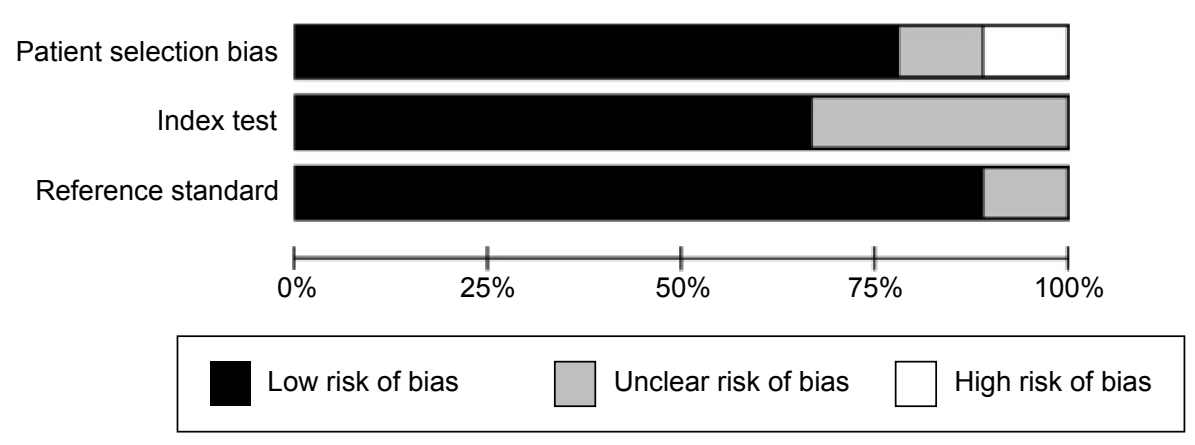

Figure 2 Overall quality assessment of included articles using the QUADAS-2 tool. Notes: (A) Risk of bias; (B) applicability concerns.

Abbreviation: QUADAS, Quality Assessment of Diagnostic Accuracy Studies.

\section{Publication bias}

In our meta-analysis, Deeks' funnel plot asymmetry test was performed to evaluate publication bias (Figure 5). The $P$-value of 0.996 suggested that no significant publication bias existed among the studies.

\section{Discussion}

Urinary cytology is the gold standard of noninvasive tests. Urinary cytology tool has low SEN. The development of high-SEN and high-SPE urinary markers for the diagnosis of urinary $\mathrm{BCa}$ is an exciting field. Currently, numerous miRNAs have been discussed in many studies. Examples of these miRNAs include miRNA-126, ${ }^{10}$ miRNA-200c, ${ }^{22}$ and miRNA ratio. ${ }^{8}$ However, the SEN and SPE of these miRNAs were uneven. Therefore, we performed this meta-analysis to provide a persuasive miRNA parameter for $\mathrm{BCa}$ diagnosis.

In the present meta-analysis, we included nine articles, including 23 studies with a total participant population of 1,213. The urine miRNAs discriminated BCa patients from healthy controls and yielded an AUC of 0.81 (95\% CI, 0.78-0.85), with pooled SEN of 0.75 (95\% CI, 0.68-0.80) and SPE of 0.75 (95\% CI, 0.70-0.80), thereby indicating the potential diagnostic value of miRNA as a noninvasive test. Furthermore, the pooled DOR was 9.07 (95\% CI,
6.35-12.95). High DOR value indicates high accuracy, thereby suggesting that the overall accuracy of miRNAs test for $\mathrm{BCa}$ diagnosis is credible.

The SROC curve, AUC, and DOR values are not the only feasible strategies for clinical diagnosis. The likelihood ratio (LR), including PLR and NLR, has an equative effect on the evaluation of diagnostic accuracy. A PLR greater than 10 or an NLR less than 0.1 generates large and often conclusive changes from pretest to posttest probability. However, in our meta-analysis, a pooled PLR of 3.03 (95\% CI, 2.50-3.67) suggested that patients with $\mathrm{BCa}$ have an $\sim 3.03$-fold higher chance of testing positive using miRNAs compared with controls. A pooled NLR of 0.33 (95\% CI, 0.27-0.42) meant that the probability of the individuals with urinary $\mathrm{BCa}$ was $33 \%$ when the miRNAs test was negative.

We selected publications in a strict manner, but potential heterogeneity was still present in our study. We found that miRNA profiling and specimen type may have partially contributed to such heterogeneity. Therefore, subgroup analyses were performed to relieve the disturbance caused by heterogeneity. Subgroups of miRNA profiling demonstrated that a combination of multiple miRNA assays (SEN, SPE, and AUC of 0.86, 0.82, and 0.91 , respectively) had a higher diagnostic performance than those of single miRNA assays (SEN, SPE, and AUC of 0.72, 
A

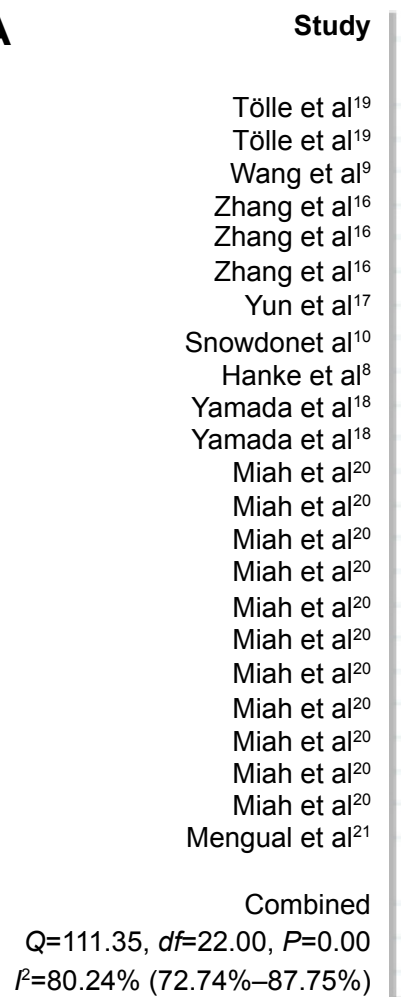

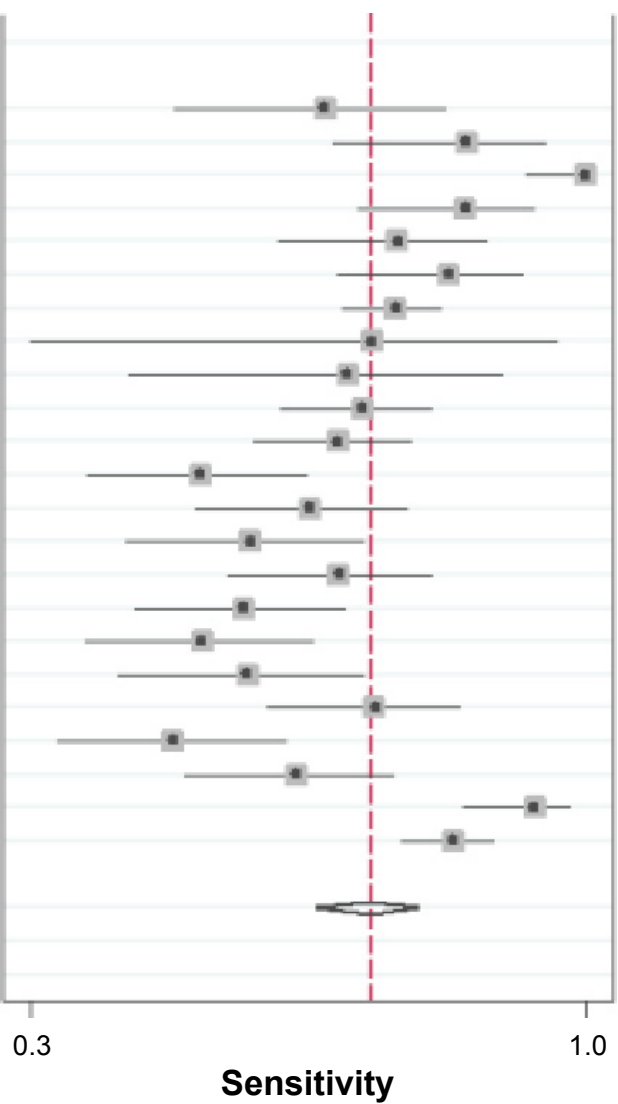

Sensitivity

B

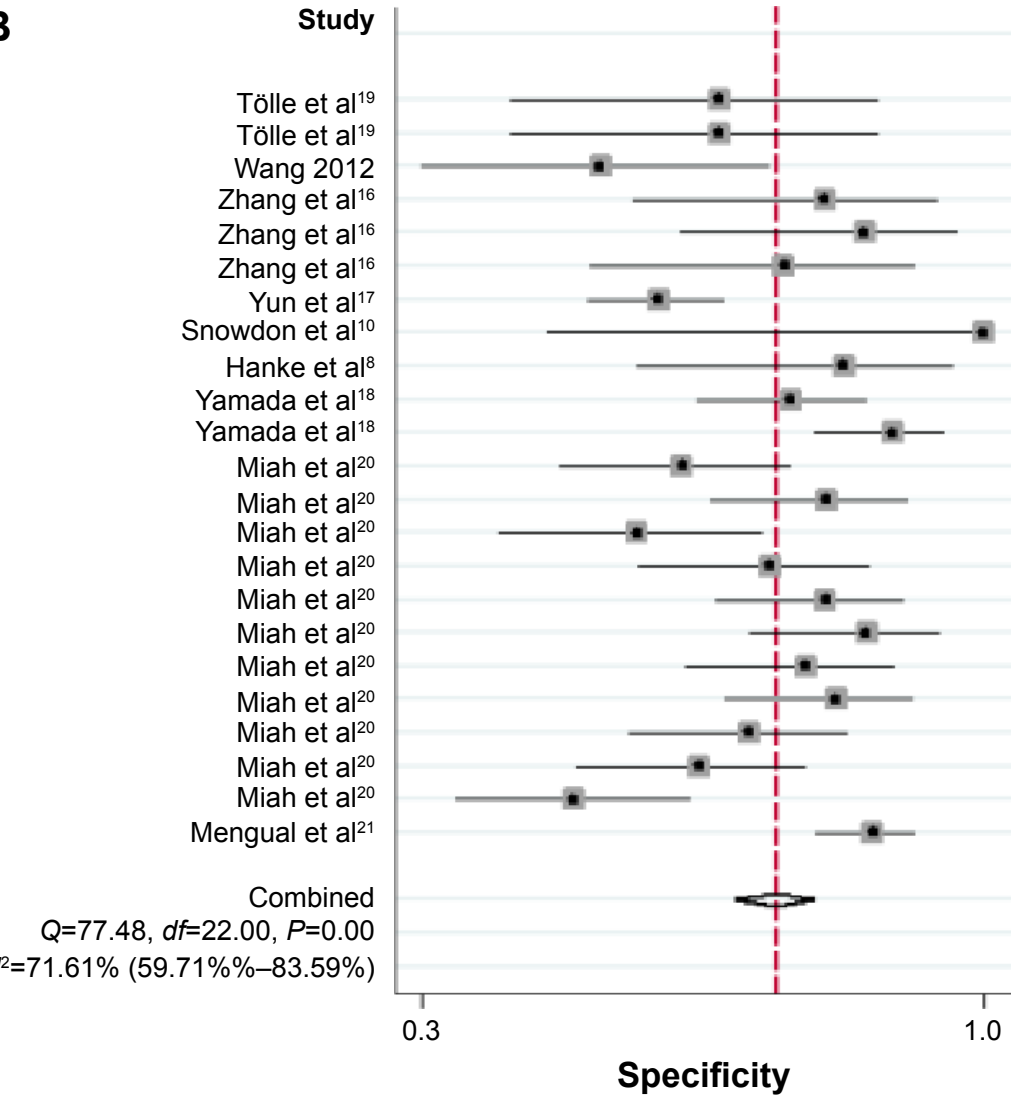

Sensitivity $(95 \% \mathrm{Cl})$

$0.69(0.52-0.84)$

$0.86(0.71-0.95)$

$1.00(0.93-1.00)$

$0.86(0.73-0.94)$

$0.78(0.64-0.88)$

$0.84(0.71-0.93)$

$0.78(0.71-0.83)$

$0.75(0.35-0.97)$

$0.72(0.47-0.90)$

$0.74(0.64-0.82)$

$0.71(0.61-0.80)$

$0.55(0.42-0.68)$

$0.68(0.54-0.79)$

$0.61(0.46-0.74)$

$0.71(0.58-0.82)$

$0.60(0.47-0.72)$

$0.55(0.42-0.68)$

$0.60(0.45-0.74)$

$0.75(0.63-0.86)$

$0.52(0.38-0.65)$

$0.66(0.53-0.78)$

$0.94(0.86-0.98)$

$0.85(0.78-0.89)$

$0.75(0.68-0.80)$
Specificity $(95 \% \mathrm{Cl})$

$0.68(0.43-0.87)$

$0.68(0.43-0.87)$

$0.54(0.33-0.74)$

$0.81(0.58-0.95)$

$0.86(0.64-0.97)$

$0.76(0.53-0.92)$

$0.61(0.53-0.69)$

$1.00(0.48-1.00)$

$0.83(0.59-0.96)$

$0.77(0.66-0.86)$

$0.89(0.80-0.95)$

$0.64(0.49-0.77)$

$0.81(0.67-0.91)$

$0.59(0.42-0.74)$

$0.74(0.59-0.86)$

$0.81(0.68-0.91)$

$0.86(0.72-0.95)$

$0.79(0.64-0.89)$

$0.82(0.69-0.92)$

$0.72(0.58-0.84)$

$0.66(0.51-0.79)$

$0.51(0.37-0.65)$

$0.87(0.80-0.92)$

$0.75(0.70-0.80)$

Figure 3 Forest plot of $(\mathbf{A})$ sensitivity and $(\mathbf{B})$ specificity of miRNAs for the diagnosis of $B C a$.

Abbreviations: $\mathrm{Cl}$, confidence interval; miRNA, microRNA; $\mathrm{BCa}$, bladder cancer. 
Table 2 Summary estimates of diagnostic criteria and their $95 \% \mathrm{Cl}$

\begin{tabular}{|c|c|c|c|c|c|c|}
\hline Subgroups & $\begin{array}{l}\text { Sensitivity } \\
(95 \% \mathrm{Cl})\end{array}$ & $\begin{array}{l}\text { Specificity } \\
(95 \% \mathrm{Cl})\end{array}$ & $\begin{array}{l}\text { Positive LR } \\
(95 \% \mathrm{Cl})\end{array}$ & $\begin{array}{l}\text { Negative LR } \\
(95 \% \mathrm{Cl})\end{array}$ & $\begin{array}{l}\text { DOR } \\
\text { (95\% Cl) }\end{array}$ & $\begin{array}{l}\text { AUC } \\
(95 \% \mathrm{Cl})\end{array}$ \\
\hline \multicolumn{7}{|l|}{ miRNA profiling } \\
\hline Single miRNA & $0.72(0.65-0.78)$ & $0.74(0.69-0.79)$ & $2.79(2.3 \mathrm{I}-3.37)$ & $0.38(0.3 \mathrm{I}-0.47)$ & $7.30(5.15-10.36)$ & $0.79(0.76-0.83)$ \\
\hline Multiple miRNAs & $0.86(0.78-0.91)$ & $0.82(0.65-0.9 \mid)$ & $4.65(2.39-9.06)$ & $0.17(0.12-0.25)$ & $27.32(14.84-50.28)$ & $0.91(0.88-0.93)$ \\
\hline \multicolumn{7}{|l|}{ Specimen } \\
\hline Voided urine & $0.77(0.66-0.84)$ & $0.76(0.63-0.85)$ & $3.12(1.98-4.91)$ & $0.31(0.21-0.47)$ & $9.99(4.7|-2| .22)$ & $0.81(0.78-0.85)$ \\
\hline Urine supernatant & $0.81(0.75-0.86)$ & $0.74(0.60-0.85)$ & $3.13(1.90-5.15)$ & $0.26(0.18-0.37)$ & $12.20(5.46-27.25)$ & $0.84(0.8 \mathrm{I}-0.87)$ \\
\hline Urine sediment & $0.73(0.63-0.8 \mathrm{I})$ & $0.75(0.69-0.8 \mathrm{I})$ & $2.96(2.34-3.73)$ & $0.36(0.26-0.49)$ & $8.20(5.17-13.03)$ & $0.81(0.77-0.84)$ \\
\hline Overall & $0.75(0.68-0.80)$ & $0.75(0.70-0.80)$ & $3.03(2.50-3.67)$ & $0.33(0.27-0.42)$ & $9.07(6.35-12.95)$ & $0.81(0.78-0.85)$ \\
\hline
\end{tabular}

Abbreviations: $\mathrm{Cl}$, confidence interval; miRNA, microRNA; DOR, diagnostic odds ratio; AUC, area under the curve; LR, likelihood ratio.

0.74 , and 0.79 , respectively). Our results were based on one miRNA that can regulate many target genes, and one target gene that could be regulated by a number of different miRNAs. ${ }^{23}$

Our results confirmed that urine miRNAs in voided urine, urine sediment, and supernatant are different. The levels of miRNAs after centrifugation can be monitored separately in the urine sediment and supernatant. ${ }^{21}$ Urine sediment generally contains renal tubular cells, normal urothelial cells, lymphocytes, red blood cells, and tumor cells. The proportion of non-BCa cells differed among urine sediments, and the obtained results can be affected by different cell types. miRNAs in urine sediment reflected intracellular expression, whereas miRNAs in urine supernatant come from microvesicles extruded from the cell surface and systemic circulation via glomerular filtration..$^{24,25}$ Therefore, compared with urinary sediment assay, urinary supernatant assay showed a relatively higher accuracy.

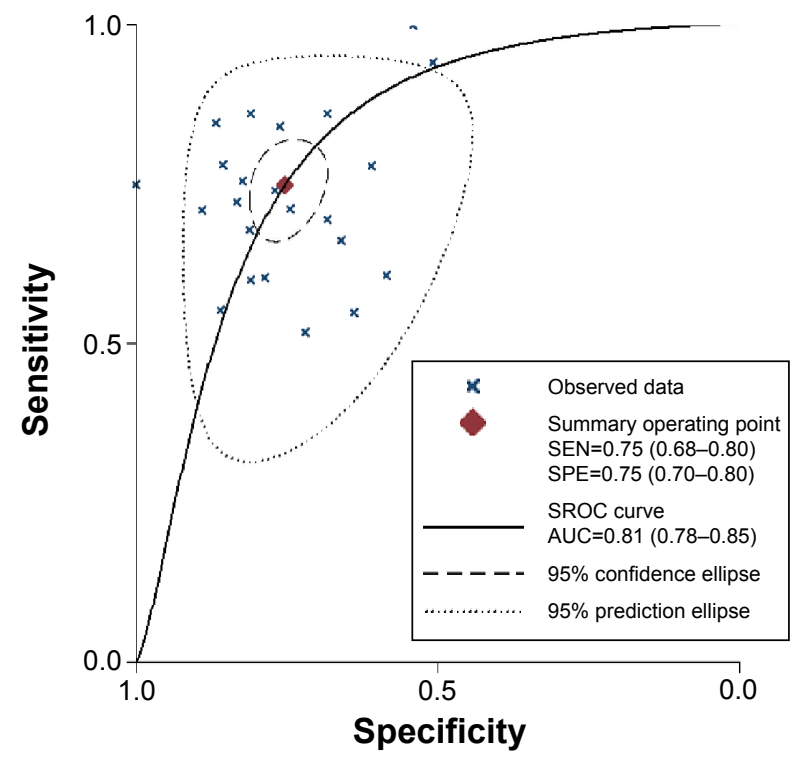

Figure 4 The SROC of the miRNA test for the diagnosis of BCa.

Abbreviations: SROC, summary receiver operating characteristic; AUC, area under the curve; BCa, bladder cancer; miRNA, microRNA; SEN, sensitivity; SPE, specificity.
This meta-analysis has several limitations. Some of the significant between-study heterogeneities were found in the current meta-analysis and may have disturbed the results. The degree of heterogeneity is among the major concerns in a metaanalysis, ${ }^{26}$ because nonhomogeneous data cause misleading results. Statistical heterogeneity among the studies was due to the differences in ethnicity, types of patients, specimens, and miRNAs profiled. Publication bias is possible even if a comprehensive and systematic search of literature was done. In addition, despite some studies identifying correlations between miRNAs expression and tumor stage and grade, subgroup analyses based on these variables were restricted due to limited reported data.

\section{Conclusion}

In this article, we analyzed and summarized the pooled data of SEN, SPE, PLR, NLR, DOR, and AUC from 23 studies. Urine miRNA assays could serve as markers for $\mathrm{BCa}$ diagnosis. We found that urine miRNA assays were more sensitive than urine cytology test in $\mathrm{BCa}$ diagnosis. However,

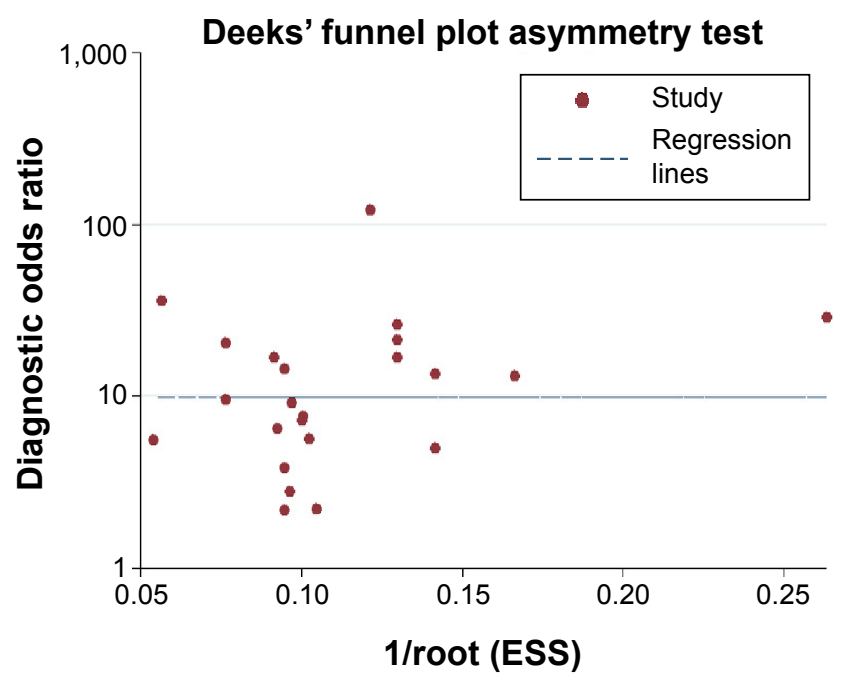

Figure 5 Deeks' linear regression test of funnel plot asymmetry. Abbreviation: ESS, effective sample size. 
the clinical application of miRNA profiling for BCa diagnosis still needs further validation by prospective studies.

\section{Acknowledgments}

This work was supported by the Program for Development of Innovative Research Team of the First Affiliated Hospital of Nanjing Medical University, the Provincial Initiative Program for Excellency Disciplines of Jiangsu Province, the National Natural Science Foundation of China (grant numbers 81272832 and 81201997), the Natural Science Foundation of Jiangsu Province (grant number BK2011848), the Priority Academic Program Development of Jiangsu Higher Education Institutions (PAPD), and Jiangsu Provincial Special Program of Medical Science (BL2012027). The funders had no role in study design, data collection and analysis, decision to publish, or preparation of the manuscript.

\section{Disclosure}

The authors report no conflicts of interest in this work.

\section{References}

1. Ploeg M, Aben KK, Kiemeney LA. The present and future burden of urinary bladder cancer in the world. World J Urol. 2009;27(3):289-293.

2. Lotan Y, Svatek RS, Sagalowsky AI. Should we screen for bladder cancer in a high-risk population? A cost per life-year saved analysis. Cancer. 2006;107(5):982-990.

3. Avritscher EB, Cooksley CD, Grossman HB, et al. Clinical model of lifetime cost of treating bladder cancer and associated complications. Urology. 2006;68(3):549-553.

4. van Rhijn BW, van der Poel HG, van der Kwast TH. Urine markers for bladder cancer surveillance: a systematic review. Eur Urol. 2005;47(6): 736-748.

5. Vrooman OP, Witjes JA. Urinary markers in bladder cancer. Euro Urol. 2008;53(5):909-916.

6. Adam L, Wszolek MF, Liu CG, et al. Plasma microRNA profiles for bladder cancer detection. Urol Oncol. 2013;31(8):1701-1708.

7. Jiang X, Du L, Wang L, et al. Serum microRNA expression signatures identified from genome-wide microRNA profiling serve as novel noninvasive biomarkers for diagnosis and recurrence of bladder cancer. Int $J$ Cancer. 2015;136(4):854-862.

8. Hanke M, Hoefig K, Merz H, et al. A robust methodology to study urine microRNA as tumor marker: microRNA-126 and microRNA-182 are related to urinary bladder cancer. Urol Oncol Semin Orig Investig. 2010;28(6):655-661.
9. Wang G, Chan ES, Kwan BC, et al. Expression of microRNAs in the urine of patients with bladder cancer. Clin Genitourin Cancer. 2012;10(2): 106-113.

10. Snowdon J, Boag S, Feilotter H, Izard J, Siemens DR. A pilot study of urinary microRNA as a biomarker for urothelial cancer. Can Urol Assoc J. 2012;15:1-5.

11. Whiting PF, Rutjes AW, Westwood ME, et al. QUADAS-2: a revised tool for the quality assessment of diagnostic accuracy studies. Ann Intern Med. 2011;155(8):529-536.

12. Deville WL, Buntinx F, Bouter LM, et al. Conducting systematic reviews of diagnostic studies: didactic guidelines. BMC Med Res Methodol. 2002;2:9.

13. Higgins JP, Thompson SG, Deeks JJ, Altman DG. Measuring inconsistency in meta-analyses. BMJ. 2003;327(7414):557-560.

14. Dinnes J, Deeks J, Kirby J, Roderick P. A methodological review of how heterogeneity has been examined in systematic reviews of diagnostic test accuracy. Health Technol Assess. 2005;9(12):1-113, iii.

15. Deeks JJ, Macaskill P, Irwig L. The performance of tests of publication bias and other sample size effects in systematic reviews of diagnostic test accuracy was assessed. J Clin Epidemiol. 2005;58(9):882-893.

16. Zhang DZ, Lau KM, Chan ES, et al. Cell-free urinary microRNA-99a and microRNA-125b are diagnostic markers for the non-invasive screening of bladder cancer. PLoS One. 2014;9(7):e100793.

17. Yun SJ, Jeong P, Kim WT, et al. Cell-free microRNAs in urine as diagnostic and prognostic biomarkers of bladder cancer. Int J Oncol. 2012; 41(5):1871-1878.

18. Yamada Y, Enokida H, Kojima S, et al. MiR-96 and miR-183 detection in urine serve as potential tumor markers of urothelial carcinoma: correlation with stage and grade, and comparison with urinary cytology. Cancer Sci. 2011;102(3):522-529.

19. Tolle A, Jung M, Rabenhorst S, Kilic E, Jung K, Weikert S. Identification of microRNAs in blood and urine as tumour markers for the detection of urinary bladder cancer. Oncol Rep. 2013;30(4):1949-1956.

20. Miah S, Dudziec E, Drayton RM, et al. An evaluation of urinary microRNA reveals a high sensitivity for bladder cancer. Br J Cancer. 2012;107(1): 123-128.

21. Mengual L, Lozano JJ, Ingelmo-Torres M, GazquezC, Ribal MJ, AlcarazA. Using microRNA profiling in urine samples to develop a non-invasive test for bladder cancer. Int $J$ Cancer. 2013;133(11):2631-2641.

22. Wszolek MF, Rieger-Christ KM, Kenney PA, et al. A microRNA expression profile defining the invasive bladder tumor phenotype. Urol Oncol. 2011;29(6):794-801.e1.

23. Wiemer EA. The role of microRNAs in cancer: no small matter. Eur J Cancer. 2007;43(10):1529-1544.

24. Wang G, Tam LS, Li EK, et al. Serum and urinary cell-free MiR-146a and MiR-155 in patients with systemic lupus erythematosus. J Rheumatol. 2010;37(12):2516-2522.

25. Wang G, Tam LS, Kwan BC, et al. Expression of miR-146a and miR-155 in the urinary sediment of systemic lupus erythematosus. Clin Rheumatol. 2012;31(3):435-440.

26. Moreno SG, Sutton AJ, Thompson JR, Ades AE, Abrams KR, Cooper NJ. A generalized weighting regression-derived meta-analysis estimator robust to small-study effects and heterogeneity. Stat Med. 2012;31(14): $1407-1417$.
OncoTargets and Therapy

\section{Publish your work in this journal}

OncoTargets and Therapy is an international, peer-reviewed, open access journal focusing on the pathological basis of all cancers, potential targets for therapy and treatment protocols employed to improve the management of cancer patients. The journal also focuses on the impact of management programs and new therapeutic agents and protocols on

Submit your manuscript here: http://www.dovepress.com/oncotargets-and-therapy-journal

\section{Dovepress}

patient perspectives such as quality of life, adherence and satisfaction. The manuscript management system is completely online and includes a very quick and fair peer-review system, which is all easy to use. Visit http://www.dovepress.com/testimonials.php to read real quotes from published authors. 